Case Report

\title{
Sodium-Glucose Cotransporter 2 Inhibitors Improve Chronic Diabetic Macular Edema
}

\author{
Yoko Takatsuna $\mathbb{D}^{1,},{ }^{1,2}$ Ryoichi Ishibashi, ${ }^{3,4}$ Tomoaki Tatsumi, ${ }^{2}$ Masaya Koshizaka, \\ Takayuki Baba, ${ }^{2}$ Shuichi Yamamoto, ${ }^{2}$ and Koutaro Yokote ${ }^{4,5}$ \\ ${ }^{1}$ Department of Ophthalmology, Chiba Rosai Hospital, Ichihara, Chiba, Japan \\ ${ }^{2}$ Department of Ophthalmology and Visual Science, Chiba University Graduate School of Medicine, Chiba, Japan \\ ${ }^{3}$ Department of Medicine, Division of Diabetes, Endocrinology and Metabolism, Kimitsu Chuo Hospital, Kisarazu, Chiba, Japan \\ ${ }^{4}$ Department of Endocrinology, Hematology and Gerontology, Graduate School of Medicine, Chiba University, Chiba, Japan \\ ${ }^{5}$ Division of Diabetes, Metabolism and Endocrinology, Chiba University Hospital, Chiba, Japan
}

Correspondence should be addressed to Yoko Takatsuna; yoko_takatsuna@chibah.johas.go.jp

Received 25 April 2020; Revised 31 August 2020; Accepted 23 October 2020; Published 16 November 2020

Academic Editor: Stephen G. Schwartz

Copyright $(2020$ Yoko Takatsuna et al. This is an open access article distributed under the Creative Commons Attribution License, which permits unrestricted use, distribution, and reproduction in any medium, provided the original work is properly cited.

\begin{abstract}
Purpose. Diabetic macular edema (DME) is a vision-threatening condition that develops in diabetic patients. The first-line therapy for DME is intravitreal injections of antivascular endothelial growth factor (anti-VEGF) agents; however, the high frequency of repeat injections, invasiveness of the procedure, and high cost are drawbacks for this treatment. The purpose of this report is to present our findings in 3 patients with chronic DME whose edema was resolved soon after oral doses of sodium-glucose cotransporter-2 (SGLT2) inhibitors were used. Case Presentation. Case 1 was a 66-year-old woman diagnosed with moderate nonproliferative diabetic retinopathy (DR) with DME that had developed a decade earlier. The DME persisted for 4 years in the left eye. The addition of oral empagliflozin, a SGLT2 inhibitor, led to a marked improvement of the DME after one month, and this improvement continued over two years. Case 2 was a 68 -year-old woman who was diagnosed with preproliferative DR with bilateral DME. The addition of oral dapagliflozin led to the improvement of the DME after two months, and this improvement continued over one year. Case 3 was a 61-year-old woman who was diagnosed with moderate nonproliferative DR with DME. Oral luseogliflozin was given which led to better glycemic control, and her left central retinal thickness (CRT) was markedly reduced after only two weeks. This reduction was maintained in her left eye for six months without any additional ophthalmic procedures. Conclusions. Although this study involved only three cases, our findings indicate that SGLT2 inhibitors might have possible efficacy for chronic DME.
\end{abstract}

\section{Introduction}

Diabetic retinopathy (DR) is a frequent cause of blindness in working-age individuals. Diabetic macular edema (DME) is one of the vision-reducing conditions that is associated with $\mathrm{DR}$, and the number of patients with DME increases with the advancement of the retinopathy.

At present, the standard treatment for DME is intravitreal injections of anti-vascular endothelial growth factor (VEGF) agents. A number of clinical studies have shown the superiority of anti-VEGF therapy over laser photocoagulation or steroid injections to treat DME $[1,2]$. However, there are some problems with the anti-VEGF therapy; the need for frequent repeat injections and the high cost of anti-VEGF agents are obstacles for their more frequent use. In addition, DME can persist even after the anti-VEGF treatment in approximately 31.6 to $65.6 \%$ of the patients [3].

Sodium-glucose cotransporter 2 (SGLT2) inhibitors are a new treatment option for patients with type 2 diabetes mellitus that improves the glycemic control by inhibiting the reabsorption of glucose in the proximal renal tubules leading to an increase in renal glucose extraction [4]. Approximately $90 \%$ of the glucose in glomerular filtrates is reabsorbed by the proximal renal tubule and returned to the blood. SGLT2 is present in the proximal renal tubule, and it cotransports glucose and sodium from the glomerular filtrate back into 

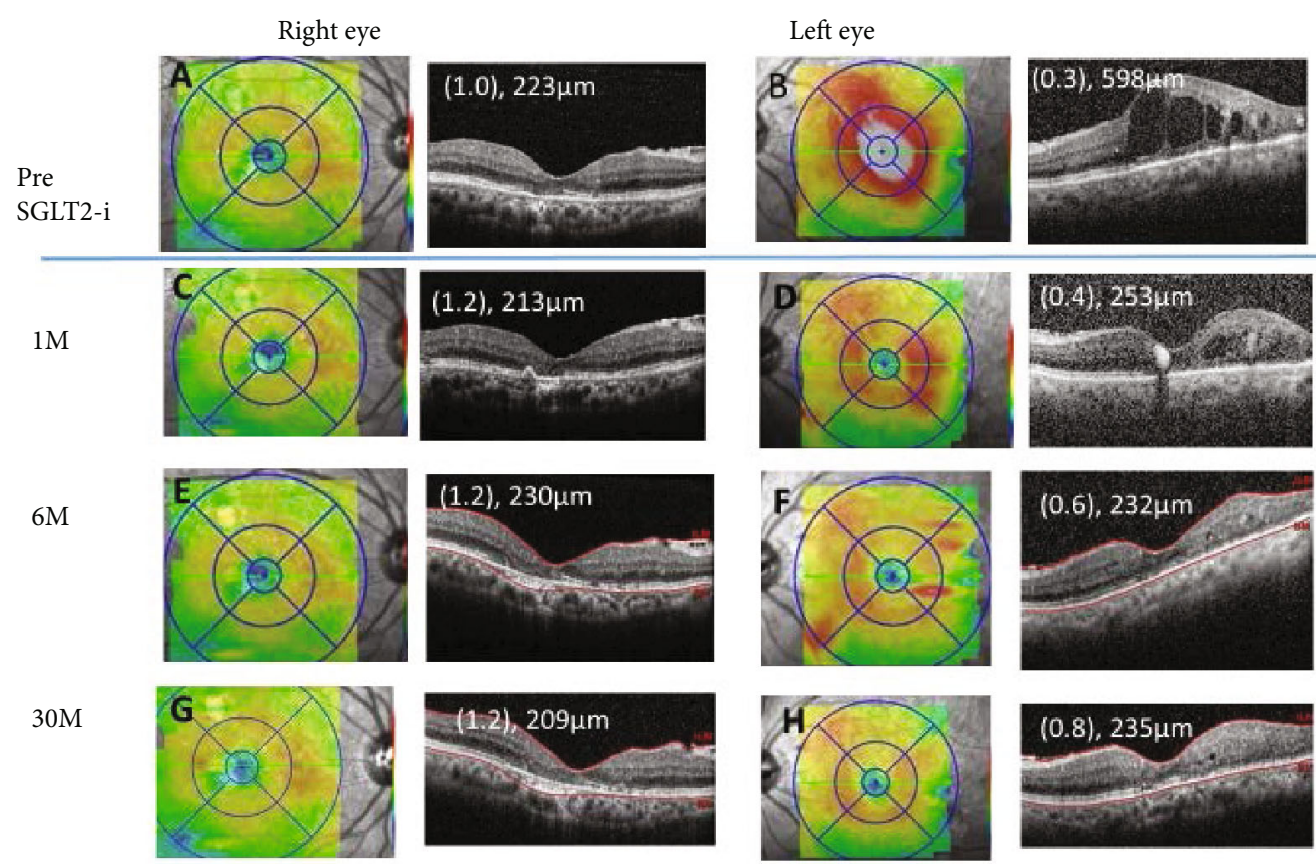

FIGURE 1: Case 1. Optical coherence tomographic images and fundus photographs showing the central retinal thickness (CRT) pre (A, B), at 1 month $(\mathrm{M})(\mathrm{C}, \mathrm{D}), 6 \mathrm{M}(\mathrm{E}, \mathrm{F})$, and $30 \mathrm{M}(\mathrm{G}, \mathrm{H})$ after commencing the oral sodium-glucose cotransporter 2 inhibitor (SGLT2i).

the blood. SGLT2 inhibitors interfere with the glucose reabsorption, and the glucose is excreted in the urine. Recent large-scale clinical trials have demonstrated that SGLT2 inhibitors prevent or reduce mortality, heart failures [5], and the progression of renal impairment [6] in diabetic patients. In addition, studies have shown the presence of SGLT2 in retinal pericytes and mesangial cells [7]. Hyperglycemia results in functional and morphological changes in these cells, but these effects are attenuated by SGLT inhibitors. Although SGLT inhibitors have been shown to be helpful in maintaining the general condition in diabetic patients, their effect on the DME in patients with DR has not been determined.

We report our findings in three patients with type 2 diabetes with chronic DME who were refractory to anti-VEGF therapies and other therapies. However, the three cases had a marked improvement of the DME soon after an oral SGLT2 inhibitor was begun. All patients had chronic DME lasting for more than 5 years and had undergone laser and/or antiVEGF and/or steroid injections.

\section{Case Presentation}

2.1. Case 1. A 66-year-old woman was diagnosed with moderate nonproliferative DR with DME bilaterally a decade earlier. She underwent focal laser photocoagulation in both eyes, and the decimal best-corrected visual acuity (BCVA) in her right eye improved to 1.0, but it did not improve in her left eye. She did not agree to further therapy such as anti-VEGF for the left eye, because the BCVA in her right eye was good and the high cost of anti-VEGF agents. The macular edema in the left eye persisted for 4 years with a decimal BCVA of 0.3 and a central retinal thickness (CRT) of $598 \mu \mathrm{m}$ (Figure 1(B)). Six months later, the macular edema was completely resolved (Figure $1(\mathrm{D})$ ). One month before this ophthalmic visit, her glycemic control deteriorated to a glycated hemoglobin A1c of 7.4\%, and her general physician prescribed $25 \mathrm{mg}$ of oral empagliflozin, an SGLT2 inhibitor. The CRT in her left eye decreased to $253 \mu \mathrm{m}$ (Figure 1(D)) at one month after beginning the SGLT2 inhibitor treatment. Six months later (Figure 1(F)), her body weight was reduced, and the glycemic control was improved with the glycated hemoglobin A1c of $6.5 \%$ (Table 1 ). The CRT reduction was maintained for over two years without other ophthalmic therapy. The clinical course of the macular edema before and after the administration of the SGLT2 inhibitor is shown in Figure 1. The final decimal BCVA improved to 0.8 , and the CRT measured by optical coherence tomography (OCT) was $235 \mu \mathrm{m}$ (Figure $1(\mathrm{H})$ ) at 30 months after commencing the SGLT2 inhibitor.

2.2. Case 2. A 68-year-old woman was diagnosed with preproliferative DR 6 years earlier, and panretinal photocoagulation had been performed. However, the DME gradually developed beginning 5 years earlier. She was treated twice with intravitreal injections of ranibizumab and a sub-Tenon injection of triamcinolone acetonide in her left eye. In her right eye, she received 7 intravitreal injections of aflibercept and focal-grid laser photocoagulation. However, the DME persisted, and her decimal BCVA was 0.6 in the right eye and 0.3 in the left eye. The CRT was $519 \mu \mathrm{m}$ in the right eye and $467 \mu \mathrm{m}$ in the left eye (Figure 2(A) and (B)). Oral dapagliflozin was administered to improve the glycemic control. At the beginning of the dapagliflozin, her glycated hemoglobin A1c was 7.0\%; however, her glycemic control was exacerbated by overconsumption; although, the refractory DME was improved. Two months later, the CRT was thinner in her left eye (Figure 2(D)), and six months later, 
TABLE 1: Summary of three cases of DME pre and 6 months (M) after the SGLT2-i administration.

\begin{tabular}{|c|c|c|c|c|c|c|}
\hline & \multicolumn{2}{|c|}{ Case 1} & \multicolumn{2}{|c|}{ Case 2} & \multicolumn{2}{|l|}{ Case 3} \\
\hline & Pre & $\begin{array}{l}6 \mathrm{M} \text { after } \\
\text { SGLT2-i }\end{array}$ & Pre & $\begin{array}{l}6 \mathrm{M} \text { after } \\
\text { SGLT2-i }\end{array}$ & Pre & $\begin{array}{l}6 \mathrm{M} \text { after } \\
\text { SGLT2-i }\end{array}$ \\
\hline Age (yrs)/sex & \multicolumn{2}{|c|}{$66 / \mathrm{F}$} & \multicolumn{2}{|c|}{$68 / \mathrm{F}$} & \multicolumn{2}{|l|}{$61 / \mathrm{F}$} \\
\hline Body height $(\mathrm{cm})$ & \multicolumn{2}{|c|}{158} & \multicolumn{2}{|c|}{156} & \multicolumn{2}{|l|}{153} \\
\hline Body weight (kg) & 49.1 & 45.2 & 43 & 41.1 & 46.1 & 45 \\
\hline Blood pressure (mmHg) & $150 / 82$ & $136 / 62$ & $128 / 70$ & $126 / 74$ & $124 / 77$ & $121 / 70$ \\
\hline SGLT2 inhibitor & - & $\begin{array}{l}\text { Empagliflozin } \\
25 \mathrm{mg}\end{array}$ & - & $\begin{array}{l}\text { Dapagliflozin } \\
5 \mathrm{mg}\end{array}$ & - & $\begin{array}{l}\text { Luseogliflozin } \\
2.5 \mathrm{mg}\end{array}$ \\
\hline Diabetes treatment & $\begin{array}{c}\text { Vildagliptin } \\
100 \mathrm{mg} \\
\text { Glimepiride } \\
1.5 \mathrm{mg}\end{array}$ & $\begin{array}{l}\text { Vildagliptin } \\
100 \mathrm{mg}\end{array}$ & $\begin{array}{l}\text { Alogliptin } 25 \mathrm{mg} \\
\text { Acarbose } 300 \mathrm{mg} \\
\text { Metformin } \\
500 \mathrm{mg}\end{array}$ & $\begin{array}{l}\text { Acarbose } \\
300 \mathrm{mg} \\
\text { Metformin } \\
500 \mathrm{mg}\end{array}$ & - & - \\
\hline Ophthalmic treatment; OD & Focal laser & - & $\begin{array}{l}\text { Focal/grid laser } \\
\quad \text { IVA } \times 7\end{array}$ & - & Focal laser, IVA $\times 1$ & - \\
\hline ; OS & Focal laser & - & $\begin{array}{c}\text { IVR } \times 2, \text { STTA } \\
\times 2\end{array}$ & - & $\begin{array}{c}\text { Focal laser } \times 3, \text { IVA } \times 5, \\
\text { STTA } \times 2\end{array}$ & - \\
\hline $\begin{array}{l}\text { Central retinal thickness } \\
(\mu \mathrm{m}) \mathrm{OD} / \mathrm{OS}\end{array}$ & $223 / 598$ & $230 / 232$ & $519 / 467$ & $491 / 326$ & $613 / 412$ & $451 / 273$ \\
\hline Visual acuity OD/OS & $(1.0) /(0.3)$ & $(1.2) /(0.6)$ & $(0.3) /(0.3)$ & $(0.6) /(0.3)$ & $(1.0) /(1.0)$ & $(1.0) /(1.0)$ \\
\hline HgAlc (\%) & 7.4 & 6.5 & 7.0 & 7.9 & 6.5 & 6.1 \\
\hline Blood glucose (mg/dL) & 116 & 118 & 118 & 129 & 113 & 100 \\
\hline Total protein $(\mathrm{g} / \mathrm{dL})$ & ND & 6.5 & 6.4 & 6.9 & 7.6 & ND \\
\hline Albumin (g/dL) & ND & ND & ND & 4.1 & 4.2 & ND \\
\hline $\begin{array}{l}\text { Aspartate aminotransferase } \\
\text { (U/L) }\end{array}$ & ND & 18 & 12 & 12 & 21 & 22 \\
\hline $\begin{array}{l}\text { Alanine aminotransferase } \\
(\mathrm{U} / \mathrm{L})\end{array}$ & ND & 13 & 11 & 11 & 18 & 18 \\
\hline Creatinine (mg/dL) & 0.47 & 0.42 & 0.61 & 0.65 & 0.59 & 0.62 \\
\hline $\begin{array}{l}\text { U-protein (qualitative } \\
\text { analysis) }\end{array}$ & - & - & - & \pm & - & - \\
\hline U-albumin (mg/g囚Cre) & 8.7 & 6.9 & ND & ND & 24.8 & ND \\
\hline
\end{tabular}

DME: diabetic macular edema; SGLT2-i: SGLT2 inhibitor; OD: oculus dexter; OS: oculus sinister; IVA: intravitreal injection of aflibercept; IVR: intravitreal injection of ranibizumab; STTA: sub-Tenon injection of triamcinolone acetonide.

the CRT was reduced to $491 \mu \mathrm{m}$ in the right eye (Figure $2(\mathrm{G})$ ) and $326 \mu \mathrm{m}$ in the left eye (Figure $2(\mathrm{H})$ ). Her BCVA was 0.6 in the right eye and 0.3 in the left. At six months, her body weight was reduced; although, the glycated hemoglobin A1c was elevated to $7.9 \%$ (Table 1). The clinical course of the macular edema is shown in Figure 2. A sub-Tenon injection of triamcinolone acetonide was administered to her right eye at eight months. Her final BCVA was 0.8 in the right eye and 0.4 in the left eye, and the CRT was $378 \mu \mathrm{m}$ in the right (Figure 2(I)), $305 \mu \mathrm{m}$ in the left (Figure 2(J)) at twelve months.

2.3. Case 3. A 61-year-old woman was diagnosed with moderate nonproliferative DR without DME 7 years earlier. She had undergone bilateral cataract surgery 5 years earlier, and her BCVA had recovered to 1.0, but there was a gradual development of macular edema. She received 2 sub-Tenon injections of triamcinolone acetonide, 5 intravitreal injections of aflibercept, and focal laser treatment in her left eye. She received 1 intravitreal injection of aflibercept and a focal laser treatment in her right eye. Despite these treatments, the CRT was still $613 \mu \mathrm{m}$ in the right eye (Figure 3(A)) and $412 \mu \mathrm{m}$ in the left eye (Figure 3(B)); although, the decimal BCVA was 1.0 in both eyes. Her BCVAs were maintained, and she did not agree to continue anti-VEGF therapy. Oral luseogliflozin $2.5 \mathrm{mg}$ was administered, and the CRT of both eyes (Figure 3(C) and (D)) began to decrease by two weeks after commencing the luseogliflozin. This reduction continued for six months. Six months later, her CRT was reduced to $451 \mu \mathrm{m}$ in the right eye (Figure 3(I)) and $273 \mu \mathrm{m}$ in the left eye (Figure $3(\mathrm{~J})$ ). The BCVA was maintained at 1.0 in both eyes. Her body weight was reduced at six months, and the glycated hemoglobin A1c was reduced from $6.5 \%$ to $6.1 \%$ at six months (Table 1).

\section{Discussion}

The findings in these three cases suggested that SGLT2 inhibitors can improve the chronic DME which was resistant to standard ophthalmic therapies. Anti-VEGF drugs are the 


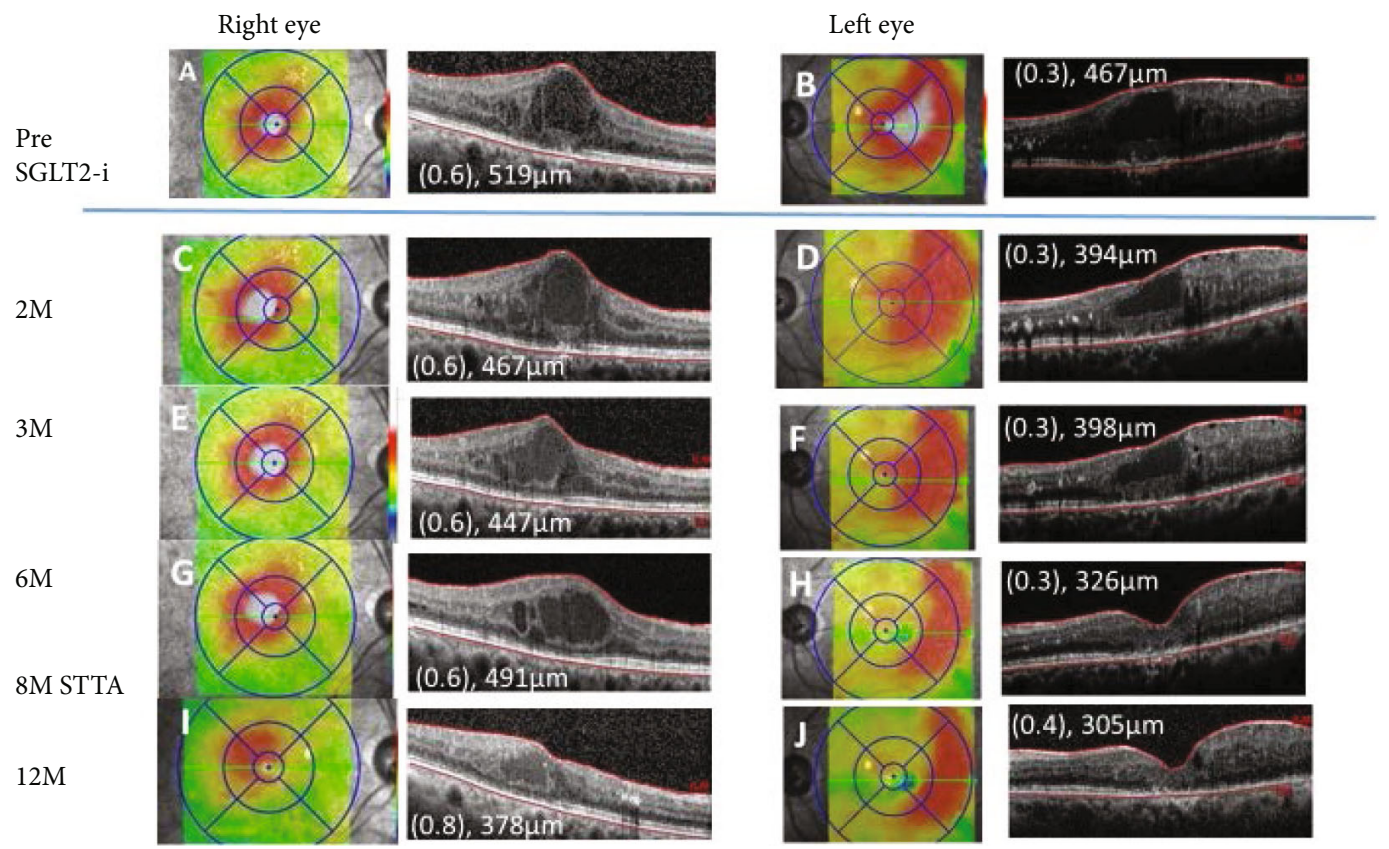

FIGURE 2: Case 2. Optical coherence tomographic images and fundus photographs showing the central retinal thickness (CRT) pre (A, B) and at 2 months $(\mathrm{M})(\mathrm{C}, \mathrm{D}), 3 \mathrm{M}(\mathrm{E}, \mathrm{F}), 6 \mathrm{M}(\mathrm{G}, \mathrm{H})$, and $12 \mathrm{M}(\mathrm{I}, \mathrm{J})$ after commencing the oral sodium-glucose cotransporter 2 inhibitor (SGLT2i). A sub-Tenon injection of triamcinolone acetonide (STTA) was administered in her right eye at eight months.

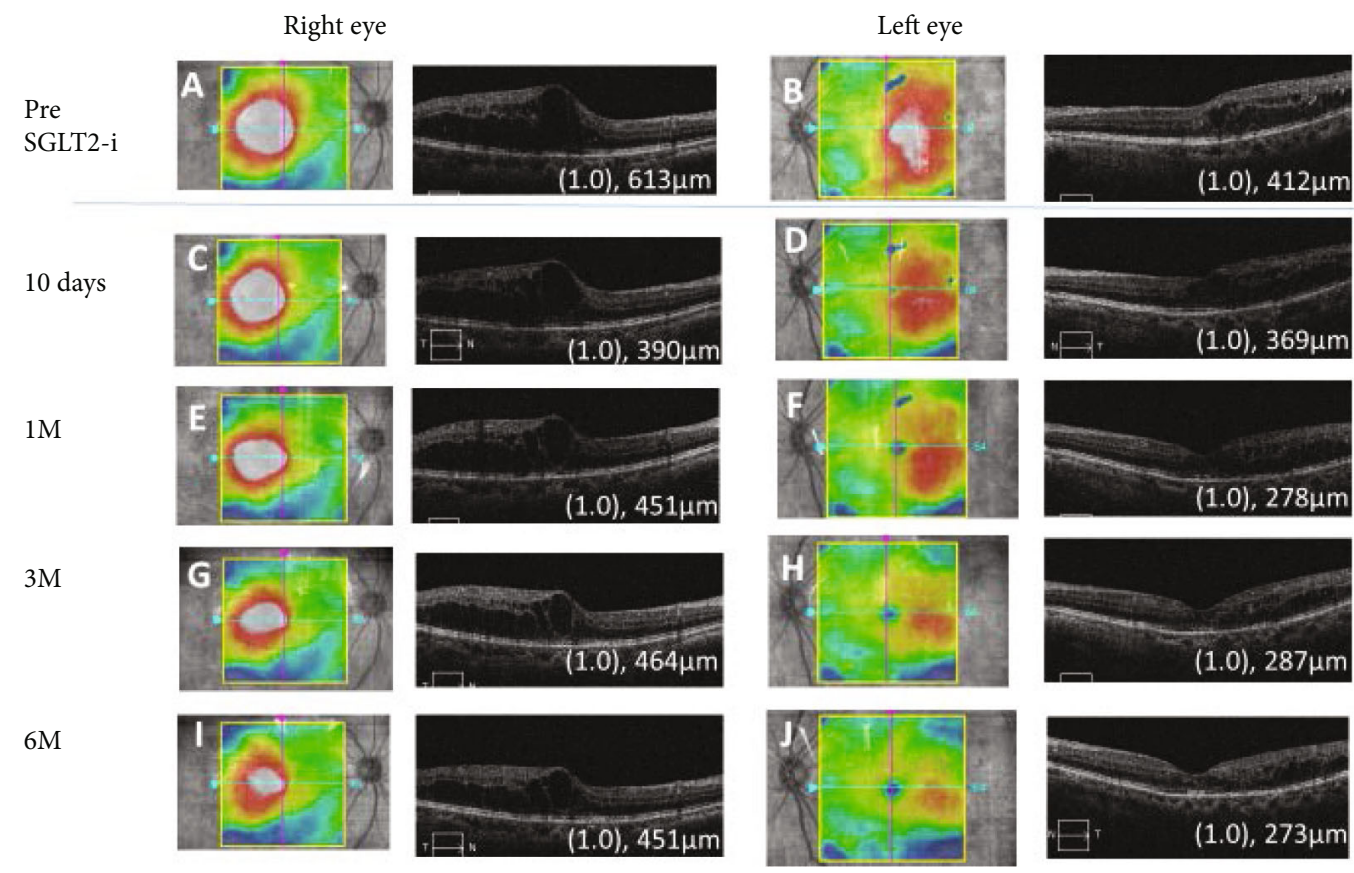

Figure 3: Case 3. Optical coherence tomographic images and fundus photographs showing the central macular thickness (CRT) pre (A, B) and at 10 days $(\mathrm{C}, \mathrm{D}), 1$ month $(\mathrm{M})(\mathrm{E}, \mathrm{F}), 3 \mathrm{M}(\mathrm{G}, \mathrm{H})$, and $6 \mathrm{M}(\mathrm{I}, \mathrm{J})$ after commencing the oral sodium-glucose cotransporter 2 inhibitor (SGLT2i).

first-line therapy for center-involved DMEs [1, 2]; however, they are costly, and clinical patients receive approximately three injections per year in Japan [8]. Our three patients did not agree to the frequent anti-VEGF injections due to their high costs, and reducing the number of anti-VEGF injections can lead to an overall decrease in the efficacy as seen in randomized trials $[1,2]$. DME pathology persisted in all three cases following the multimodal therapy including steroid injections and focal/grid laser photocoagulation in our cases. However, stabilization and recovery were observed soon after the oral administration of a SGLT2 inhibitor. Notably, the improvements were detected as soon as two 
weeks after the initiation of the SGLT2 inhibitor in Case 3, and the improvements were maintained for at least 2 years in Case 1.

There is no evidence that internal medications have positive effects on DME, but two studies have reported the effectiveness of SGLT2 inhibitors for the treatment of DME [9, 10]. One study reported on the efficacy of SGLT2 inhibitors on the vitrectomized eyes of five patients [9]. However, vitrectomy is not the standard or even the preferential treatment for DME, and anti-VEGF therapy or other standard treatments were omitted in that study. An additional study presented a case of a DME patient who was given ipragliflozin. The patient was not treated with any anti-VEGF before the SGLT2-inhibitor administration, and OCT showed no significant changes after 24 weeks of SGLT2-inhibitor administration [10].

The precise mechanism by which SGLT2 inhibitors resolve the DME was not determined. Weight loss was observed in all cases, and the rapid effects of the SGLT2 inhibitors may be related to its diuretic effects. In Case 2, however, the blood glycemic level did not improve, suggesting that a short period of glycemic control improvement by SGLT2 inhibitors may not have a direct effect on the DME.

Empagliflozin reduces the risk of major adverse cardiovascular events [5] and reduces the progression of kidney diseases [6]. SGLT2 inhibitors may improve DME via systemic fluid adjustments.

One in vitro study reported that phlorizin, an SGLT inhibitor, attenuated the high glucose-induced morphologi$\mathrm{cal}$ and functional changes in cultured bovine retinal pericytes [11].The expression of SGLT1 and SGLT2 in the eye including the retina has been reported in several papers [7, 12]. SGLT2 inhibitors remove excess glucose from the retinal microcirculation and hence reduce glucotoxicity, oxidative stress, low-grade inflammation, and restore insulin signaling. By preventing continued glucose-induced vascular dysfunction, the progression of microangiopathy and especially DR is improved $[13,14]$. The long-lasting effect of SGLT2 inhibitors might be related to the reduction of the glucose-induced vascular endothelial dysfunction.

It has been well established that anti-VEGF therapy is the mainstay treatment for DME, and oral SGLT2 inhibitors combined with anti-VEGF injections may be additive and a good option for refractory DME.

There are some limitations in this study. Only three cases with widely different histories were studied, and all cases were undertreated with anti-VEGF agents compared to the standardized regimens. Additionally, three different SGLT2 inhibitors were prescribed to the three patients; all were effective in reducing the degree of DME, but their mechanism of action has not been clearly determined.

Therefore, we are planning a multicenter, randomized, open-label trial to investigate the safety and efficacy of combination therapy of anti-VEGF drug injections and SGLT2 inhibitors in patients with type 2 diabetes and DME [15].

In conclusion, our results showed an improvement of chronic DME after the initiation of oral SGLT2 inhibitors. SGLT2 inhibitors might be a novel, noninvasive, and lowcost treatment for DME.

\section{Conflicts of Interest}

None of the authors has conflicts of interest with this submission.

\section{Acknowledgments}

The authors thank Professor Emeritus Duco Hamasaki of the Bascom Palmer Eye Institute for discussions and for editing the manuscript.

\section{References}

[1] The Diabetic Retinopathy Clinical Research Network, "Aflibercept, bevacizumab, or ranibizumab for diabetic Macular edema," New England Journal of Medicine, vol. 372, no. 13, pp. 1193-1203, 2015.

[2] P. Mitchell, F. Bandello, U. Schmidt-Erfurth et al., "The RESTORE study: ranibizumab monotherapy or combined with laser versus laser monotherapy for diabetic macular edema," Ophthalmology, vol. 118, no. 4, pp. 615-625, 2011.

[3] N. M. Bressler, W. T. Beaulieu, A. R. Glassman et al., "Persistent macular thickening following intravitreous aflibercept, bevacizumab, or ranibizumab for central-involved diabetic macular edema with vision impairment: a secondary analysis of a randomized clinical trial," JAMA Ophthalmology, vol. 136, no. 3, pp. 257-269, 2018.

[4] G. E. Dailey, "Empagliflozin: a new treatment option for patients with type 2 diabetes mellitus," Drugs of Today, vol. 51, no. 9, pp. 519-535, 2015.

[5] B. Zinman, C. Wanner, J. M. Lachin et al., "Empagliflozin, cardiovascular outcomes, and mortality in type 2 diabetes," New England Journal of Medicine, vol. 373, no. 22, pp. 2117-2128, 2015.

[6] C. Wanner, S. E. Inzucchi, J. M. Lachin et al., "Empagliflozin and progression of kidney disease in type 2 diabetes," New England Journal of Medicine, vol. 375, no. 4, pp. 323-334, 2016.

[7] M. Wakisaka and T. Nagao, "Sodium glucose cotransporter 2 in mesangial cells and retinal pericytes and its implications for diabetic nephropathy and retinopathy," Glycobiology, vol. 27, no. 8, pp. 691-695, 2017.

[8] M. Sugimoto, H. Tsukitome, F. Okamoto et al., "Clinical preferences and trends of anti-vascular endothelial growth factor treatments for diabetic macular edema in Japan," Journal of Diabetes Investigation, vol. 10, no. 2, pp. 475-483, 2019.

[9] H. Mieno, K. Yoneda, M. Yamazaki, R. Sakai, C. Sotozono, and M. Fukui, "The efficacy of sodium-glucose cotransporter 2 (SGLT2) inhibitors for the treatment of chronic diabetic macular oedema in vitrectomised eyes: a retrospective study," BMJ Open Opthalmology, vol. 3, no. 1, article e000130, 2018.

[10] H. Yoshizumi, T. Ejima, T. Nagao, and M. Wakisaka, "Recovery from diabetic macular edema in a diabetic patient after minimal dose of a sodium glucose co-trasporter 2 inhibitor," American Journal of Case Reports, vol. 19, pp. 462-466, 2018.

[11] M. Wakisaka, M. Yoshinari, T. Asano et al., "Normalization of glucose entry under the high glucose condition by phlorizin attenuates the high glucose-induced morphological and functional changes of cultured bovine retinal pericytes," Biochimica et Biophysica Acta, vol. 1453, no. 1, pp. 83-91, 1999.

[12] L. Y. Herat, V. B. Matthews, P. E. Rakoczy, R. Carnagarin, and M. Schlaich, "Focusing on sodium glucose cotransporter-2 and 
the sympathetic nervous system: potential impact in diabetic retinopathy," International Journal of Endocrinology, vol. 2018, Article ID 9254126, 8 pages, 2018.

[13] M. Oelze, S. Kröller-Schön, P. Welschof et al., "The sodiumglucose co-transporter 2 inhibitor Empagliflozin improves diabetes-induced vascular dysfunction in the streptozotocin diabetes rat model by interfering with oxidative stress and glucotoxicity," PLoS One, vol. 9, no. 11, article e112394, 2014.

[14] M. May, T. Framke, B. Junker, C. Framme, A. Pielen, and C. Schindler, "How and why SGLT2 inhibitors should be explored as potential treatment option in diabetic retinopathy: clinical concept and methodology," Therapeutic Advances in Endocrinology and Metabolism, vol. 10, 2019.

[15] R. Ishibashi, Y. Takatsuna, M. Koshizaka et al., "Safety and efficacy of ranibizumab and luseogliflozin combination therapy in patients with diabetic macular edema: protocol for a multicenter, open-label randomized controlled trial," Diabetes Therapy, vol. 11, no. 8, pp. 1891-1905, 2020. 